Running head: MIRANDA-FUENTES et al.

\title{
Evidence of soil-located competition as the cause of the reduction of sunflower verticillium wilt by entomopathogenic fungi
}

Pedro Miranda-Fuentes ${ }^{1,2}$, Ana Belén García-Carneros ${ }^{1}$, Ana María MontillaCarmona $^{1}$, Leire Molinero-Ruiz ${ }^{1}$

${ }^{1}$ Institute for Sustainable Agriculture, CSIC. Alameda del Obispo s/n, 14004, Cordoba, Spain ${ }^{2}$ Department of Agronomy, ETSIAM, University of Cordoba, Campus de Rabanales. Edificio C4 Celestino Mutis, 14071, Cordoba, Spain

\section{Correspondence}

L. Molinero-Ruiz; e-mail: lmolinero@ias.csic.es

Keywords

biological control, entomopathogens, integrated pest management, plant pathogens, soilborne pathogens, sunflower diseases

The increasing limitation of agrochemicals for disease control is a major challenge for European agriculture and a spur to developing environmentally friendly approaches such as biological control. Entomopathogenic fungi, which have been used in the control of insect pests for a long time, also have other uses, such as being antagonists of fungi, including plant pathogens. We determined the in vitro effect of three strains of Metarhizium brunneum and 
two of Beauveria bassiana against Verticillium dahliae and Cadophora helianthi, causal agents of sunflower wilts. Both M. brunneum and B. bassiana were able to inhibit the mycelial growth of the sunflower pathogens and, according to the dual culture and microscopy results, two types of antagonism were observed as being dependent on the strain: competition and/or antibiosis. Greenhouse experiments showed that, after soil treatments with entomopathogens and plant inoculation by root immersion in conidial suspensions of $V$. dahliae, the entomopathogens were able to efficiently persist in the soil, and three of the four strains even significantly reduced the severity of symptoms in sunflowers. Interestingly, molecular analysis showed that all the strains were able to establish themselves as endophytes in sunflowers in the absence of $V$.dahliae. When the plants were inoculated with $V$. dahliae, we detected the pathogen, but not the entomopathogen, in the sunflowers by molecular methods. The results of this work suggest that the protection conferred by M. brunneum and B. bassiana against verticillium wilt might not be plant-located, but is probably the consequence of their competition with $V$. dahliae in the soil.

\section{Introduction}

Sunflower (Helianthus annuus) is the fourth largest oilseed crop worldwide. It is grown across all five continents but is particularly relevant in Europe, where over $20 \%$ of the world's production is located (FAOSTAT, 2020). Sunflower production is constrained worldwide by diseases. Although yield decreases largely depend on the pathogen, losses of up to $100 \%$ are frequently recorded when infections are severe (Gulya et al., 2019). Several sunflower pathogens have been described, but only about a dozen of them are important in economic terms (Gulya et al., 1997). 
Verticillium dahliae, the causal agent of verticillium wilt and leaf mottle, is a soilborne ascomycete and a well-known vascular pathogen of several crop species that has been described in more than 200 different hosts (Pegg and Brady, 2002). Some of the most important hosts in economic terms are tomato, tobacco, potato, lettuce, cotton, olive tree, eggplant, artichoke, cauliflower, and sunflower. This pathogen is able to persist in the soil via microsclerotia for up to 14 years, affecting the yield of subsequent crops (Pegg and Brady, 2002). Yield losses due to $V$. dahliae are variable and largely depend on the affected crop, so that losses may range from $10 \%$ to $100 \%$ (Gulya et al., 1997; Klosterman et al., 2009), which makes this fungus one of the most important pathogens in many of its hosts, including sunflower (Martin-Sanz et al., 2018b).

The management of verticillium wilt is highly dependent on the host plant, and none of the available alternatives provide complete control of the pathogen (Pegg and Brady, 2002). Chemical control of verticillium wilt is based on fumigation or soil amendments with chemical (e.g., propamocarb) or organic (proteins, volatile fatty acids) compounds, yet this is only useful in certain crops (e.g., strawberry or pepper) and is limited by legal, economic, and/or environmental constraints (Rekanovic et al., 2007; Klosterman et al., 2009). Biological control, carried out by using fungi (a wide range of genera including Fusarium spp., Phoma spp., and many others) or bacteria (mainly pseudomonads), has been addressed by many authors (Varo et al., 2016), although none of the proposed methods have so far proven to be effective for disease control in field applications (Klosterman et al., 2009). Genetic resistance to verticillium wilt has been described and is widely used in crops like alfalfa, cotton, potato, tomato, strawberry, sunflower, oilseed rape, and lettuce (Klosterman et al., 2009).

In the case of sunflower, $V$. dahliae is one of the most important pathogens and is widespread in the USA, Argentina, Europe (Pegg and Brady, 2002; Gulya et al., 2019; 
Molinero-Ruiz, 2019), and some regions of Canada (Erreguerena et al., 2019). The only control measure available in sunflower is genetic resistance (Pegg and Brady, 2002), which was first reported in this crop in Manitoba in 1957 (Putt, 1958) and was found to be race specific and based on single genes (Fick and Zimmer, 1974). Since then, and due to further breeding and genetics programmes, resistance to verticillium wilt has been widely explored in sunflower, and several cultivars with resistance to the main pathogenic races have been obtained or identified, including new resistance sources (Radi and Gulya, 2007). However, the appearance of new races of $V$. dahliae that overcome the known resistance sources in the USA, Argentina, and Spain greatly threatens this disease control method (Gulya, 2007; Garcia-Ruiz et al., 2014).

Despite the undeniable importance of $V$. dahliae, there are recent reports of emerging sunflower diseases that can threaten the crop in some cultivation areas (Martin-Sanz et al., 2018a). Cadophora helianthi is of particular importance in Ukraine and Russia, where $26 \%$ and $21 \%$, respectively, of the world's sunflower oil is produced (FAOSTAT, 2020). Initially, C. helianthi was mistakenly identified as C. malorum (Martin-Sanz et al., 2018a), but later it was reported as a new species with special morphological and molecular traits (Crous et al., 2019). Because no reports on C. helianthi existed until 2018, no control measures are yet available. Moreover, its geographic distribution and economic importance remain unknown, although it has been associated with increasing incidence of sunflower wilt in Russia (MartinSanz et al., 2018a).

Traditionally, chemical control of plant diseases has relied heavily on synthetic chemical compounds. However, these are not effective against certain diseases including verticillium wilt (Klosterman et al., 2009). Furthermore, chemical treatments can induce the resistance of the pathogen to active ingredients (Molinero-Ruiz et al., 2003; 2008). Biological control is a topical option for effective disease management and, within it, entomopathogenic 
fungi $(\mathrm{EF})$ are a feasible alternative. The latter are pathogenic to insects, are naturally present in a wide number of environments, and are often associated with plants, via the rhizosphere or even as plant endophytes or epiphytes (Garrido-Jurado et al., 2015). Certain EF have proved to be useful tools for controlling many insect pests within integrated pest management (IPM) strategies (Quesada-Moraga et al., 2014). Thus, more than 170 fungal strains, most of which belong to Beauveria bassiana and Metarhizium anisopliae, are registered worldwide (de Faria and Wraight, 2007). Their efficacy lies both in the mortality caused by the mechanical damage produced during the propagation of fungal structures through the insect's organs and haemolymph, and in the secretion of insecticidal compounds (Quesada-Moraga et al., 2014).

However, EF can play other ecological roles, such as promoting plant growth (e.g., Metarhizium brunneum and B. bassiana promoting sorghum and sunflower growth; RayaDiaz et al., 2017a, 2017b); inducing systemic resistance (e.g., B. bassiana eliciting defensive responses in tomato; Shrivastava et al., 2015); producing compounds used in the pharmaceutical industry (e.g., terpenoids, steroids, and phenols; Schulz et al., 2002); enhancing plant nutrition (e.g., M. brunneum and B. bassiana improving Fe nutrition in sorghum and sunflower; Raya-Diaz et al., 2017a, 2017b); and as antagonists of other fungi (Ownley et al., 2010; Keyser et al., 2016). Recent works have reported some EF as acting as antagonists of plant soilborne pathogens affecting, among others, olive and wheat, both in vitro (e.g., M. brunneum and B. bassiana inhibiting Phytophthora inundata; Lozano-Tovar et al., 2013) and in vivo (e.g., Clonostachys rosea and Metarhizium spp. reducing wheat infection by Fusarium culmorum; Keyser et al., 2016). The mechanisms underlying the antagonism between EF and other fungi are mainly associated with nutrient competition and antimicrobial metabolite production (Lozano-Tovar et al., 2013, 2017), although the nature of those metabolites has not yet been explored. In sunflower, better vegetative growth and 
nutrient absorption due to the inoculation with EF was reported (Raya-Diaz et al., 2017a), as well as a successful control of two sunflower insect pests: the maize leaf weevil Tanymecus dilaticollis (Coleoptera: Curculionidae; Takov et al., 2013), and the banded sunflower moth Cochylis hospes (Lepidoptera: Tortricidae; Barker, 1999). However, EF interactions with sunflower pathogens have not been studied so far.

The aim of this study was to determine whether five strains of two different EF species (three strains of M. brunneum and two of B. bassiana) could be suitable candidates as biological control agents against the sunflower pathogens $V$. dahliae and $C$. helianthi. Thus, we carried out both in vitro experiments, in which the EF were dually plated against both pathogens, and in planta ones, in which we monitored the effect of the EF on the severity of verticillium wilt symptoms in sunflowers. We also assessed the time lapse of EF in the substrate and performed a molecular detection of the fungi in the plants.

\section{Materials and methods}

\subsection{Fungal isolates}

All the isolates of the sunflower pathogens $V$. dahliae and C. helianthi and EF M. brunneum and B. bassiana included in this study are shown in Table 1. The five EF strains tested were selected from the culture collection in the Agricultural Entomology laboratory of the Department of Agronomy, University of Cordoba (Spain) and are deposited in the Spanish Collection of Culture Types at the University of Valencia (Spain) (Raya-Diaz et al., 2017a). These strains were selected on the basis of their efficacy against insect pests or plant pathogens (Lozano-Tovar et al., 2013) and their previous use in sunflower (Raya-Diaz et al., 2017a). 
All $V$. dahliae isolates used were previously characterized in a multidisciplinary study (Martin-Sanz et al., 2018b) and were selected on the basis of their different geographical origin and pathogenic race (Garcia-Carneros et al., 2014; Martin-Sanz et al., 2018b). Cadophora helianthi isolates were selected from the plant pathogen culture collection in the Field Crop Disease Laboratory at the Institute for Sustainable Agriculture from CSIC (IASCSIC) in Cordoba, Spain (Molinero-Ruiz, 2019).

\subsection{Dual cultures}

Dual cultures were carried out to challenge the sunflower pathogens $V$. dahliae and $C$. helianthi against five strains of the EF B. bassiana and M. brunneum (Table 1). Active cultures were obtained on fresh potato dextrose agar (PDA; BD) by streaking a loop followed by incubation at $25{ }^{\circ} \mathrm{C}$ in the dark. Pieces $\left(2 \mathrm{~mm}^{2}\right)$ of actively growing seven-day-old colonies of either $V$. dahliae or $C$. helianthi, and EF, were dually plated on malt extract agar (MA; Scharlab S. L.) at a distance of $3 \mathrm{~cm}$ and incubated under the aforementioned conditions. The dual cultures were evaluated until the control treatment colonies reached the edge of the plate, 37 days after plating. The growth of every colony was recorded periodically by directly drawing its contour on the plate. After the last evaluation, photographs of every plate were taken. Pictures were analysed with the free software GIMP v. 2.8.22 (https://www.gimp.org/). The percentage of the mycelial growth inhibition (IMG) was calculated from the last measurement, corresponding to the definitive growth over the plate. The IMG was expressed as:

$\mathrm{IMG}=100 \times \frac{\text { Colony diameter in the control }- \text { Colony diameter in the treatment }}{\text { Colony diameter in the control }}$.

Experiments were carried out, independently, for $V$. dahliae and C. helianthi, in completely randomized factorial designs with three replications (plates) for $V$. dahliae and four for $C$. helianthi, the EF strains and fungal isolates being the two factors. 


\subsection{Hyphal interaction}

Interactions between hyphae of both the sunflower pathogens and the EF were observed under the microscope. For this purpose, a sterile microscope slide was covered with a thin layer of MA and isolates VdS0113 of $V$. dahliae or CadoSU01-17 of C. helianthi (Table 1), and the EF strains were placed dually at a distance of $3 \mathrm{~cm}$ perpendicular to the slide. The slides were incubated at $25{ }^{\circ} \mathrm{C}$ in the dark and monitored until the growth front of both fungi was perceived. They were then observed under a microscope.

\subsection{Greenhouse experiment}

An experiment was conducted to assess the effect of EF on the development of verticillium wilt symptoms in sunflower under greenhouse conditions. According to the results obtained in the dual culture assays, all the EF isolates in Table 1, except EAMb 01/158-Su (which did not significantly inhibit any of the pathogens), were included in the experiment. The isolate VdS0113 of $V$. dahliae, whose pathogenicity against sunflower was tested in our previous works (Gonzalez-Fernandez, 2015; Martin-Sanz et al., 2018b), and the sunflower breeding line RHA801, genetically susceptible to $V$. dahliae (Gonzalez-Fernandez, 2015), were used.

Sunflower seeds were surface-sterilized by a 10 -min immersion in $10 \%$ sodium hypochlorite, rinsed twice with sterile deionized water and put into Petri dishes containing a thin layer of water-saturated perlite covered with sterile filter paper. Seeds were incubated in darkness at $25^{\circ} \mathrm{C}$ for $48 \mathrm{hr}$ until radicles $2-5 \mathrm{~mm}$ long developed. Then, the seedlings were transplanted into high density polyethylene trays $(19.5 \times 26.5 \times 6.5 \mathrm{~cm})$ containing sterile vermiculite (one treatment per tray). Each tray was watered with $100 \mathrm{ml}$ tap water and then maintained in a greenhouse at $24{ }^{\circ} \mathrm{C}$ and a $14 \mathrm{hr}$ photoperiod for $48 \mathrm{hr}$ until treatments with EF were carried out on plantlets at the VE development stage (Schneiter and Miller, 1981), four days after sowing (DAS). 
The EF strains were applied similarly to the method described by Raya-Diaz et al. (2017b). They were plated in MA and incubated in the dark at $25^{\circ} \mathrm{C}$ for $10-15$ days. Fungal suspensions were prepared by scraping the mycelium with a sowing handle and suspending the fungus in $5 \mathrm{ml}$ sterile deionized water. The suspensions were filtered through several layers of sterile cheesecloth to retain the mycelium, and homogenized by vortex.

Concentrations were adjusted to $10^{8}$ conidia/ml using a Neubauer chamber (Blau Brand). Each treatment (i.e., four plants; each plant a replication) was watered with $100 \mathrm{ml}$ of the corresponding fungal suspension. Control plants were watered with $100 \mathrm{ml}$ sterile deionized water. Plants were grown in the greenhouse under the aforementioned conditions for 1 month. During this time, they were given a nutrient solution (Hoagland and Arnon, 1950), $100 \mathrm{ml} /$ plant, once a week and watered as needed.

Sunflowers were inoculated with $V$. dahliae 1 month after the treatment with the EF, 34 DAS, when plants were at V6 stage (Schneiter and Miller, 1981). Inoculation was carried out according to Martin-Sanz et al. (2018b) by manually uprooting the plants and immersing their root systems in $10^{6}$ conidia/ml suspensions of $V$. dahliae, prepared as described above, for $30 \mathrm{~min}$. Similarly, control plants were uprooted and immersed in sterile deionized water. After the inoculation, plants were individually transplanted into $0.7 \mathrm{~L}$ pots containing a mixture of sand:silt:peat $(2: 1: 4)$ and incubated in the greenhouse under the aforementioned conditions for 1 month. The experiment was carried out in a completely randomized factorial design with four replications (plants), the two factors being $V$. dahliae (inoculated and noninoculated control) and EF (four strains and nontreated control). The severity of symptoms (Ss), expressed as the percentage of foliar tissue showing symptoms of wilting (Garcia-Ruiz et al., 2014), was assessed weekly for each plant until the end of the experiment, 4 weeks after inoculation with the pathogen and 64 DAS. By then, plants were at R5 (Schneiter and Miller, 1981). The sequential values were used to calculate the area under 
the disease progress curve (AUDPC) by the trapezoidal integration method (Campbell and Madden, 1990).

The population density of the EF isolates inside the pots was assessed weekly, from the day on which plants were inoculated with $V$. dahliae (34 DAS) until the end of the experiment (64 DAS) in accordance with the protocol described by Raya-Diaz et al. (2017a) with slight modifications: briefly, a sample of $3 \mathrm{~g}$ substrate was collected at a depth of $0-3$ $\mathrm{cm}$ randomly from the four replications (plants) of each EF treatment noninoculated with $V$. dahliae and suspended in $30 \mathrm{ml}$ sterile deionized water and then shaken with an orbital shaker at $120 \mathrm{rpm}$ for $90 \mathrm{~min}$ (Raya-Diaz et al., 2017a). The suspensions were diluted 10-fold in sterile deionized water and $100 \mu 1$ aliquots were plated on Sabouraud dextrose agar, supplemented with $0.5 \mathrm{~g} / \mathrm{L}$ chloramphenicol (SDAC; Scharlab, S. L.). Plates (four replicates for each treatment) were incubated at $25^{\circ} \mathrm{C}$ for 5-7 days. Colonies identified as M. brunneum or B. bassiana were counted and cfu per g of substrate were calculated for each EF strain and replication.

\subsection{Microbiological and molecular detection of $V$. dahliae and entomopathogenic fungi in sunflower}

The plant colonization of both $V$. dahliae and EF was assessed at the end of the greenhouse experiment, when the fourth and last evaluation of Ss was done, 64 DAS. For $V$. dahliae, we used the methodology described by Martin-Sanz et al. (2018b): briefly, 2-cm long fragments of the stem base of each plant were cut and surface-sterilized by a $10 \mathrm{~min}$ immersion in $10 \%$ sodium hypochlorite, then rinsed twice and dried under sterile air flow. The fragments were divided into $2-5 \mathrm{~mm}$ pieces, which were plated on PDA and incubated at $25{ }^{\circ} \mathrm{C}$ for $5-7$ days. For the EF, we performed the isolation according to Gonzalez-Mas et al. (2019): leaves were removed, sterilized by a 2 min immersion in 1\% sodium hypochlorite, rinsed twice and dried 
under sterile air flow. Then, fragments of $2 \mathrm{~cm}^{2}$ were cut, plated on SDAC and incubated at $25{ }^{\circ} \mathrm{C}$ for $5-7$ days.

For molecular diagnostic analyses, also at the end of the experiment 64 DAS, we surface-sterilized stem tissues as described above and lyophilized them. Total genomic DNA from lyophilized stem tissues of all the plants (noninoculated and nontreated controls, and plants only treated with the entomopathogen, only inoculated with $V$. dahliae, or both treated with the entomopathogen and inoculated with $V$. dahliae) was individually purified using the i-genomic Plant DNA Extraction NucleoSpin Plant II (Macherey-Nagel GmbH and Co. KG) according to the manufacturer's instructions. The quality and concentration of DNA samples were determined with a Qubit 3.0 fluorometer (Invitrogen). Finally, DNA samples were adjusted to a final concentration of $10 \mathrm{ng} / \mu 1$ and stored at $-20{ }^{\circ} \mathrm{C}$ until required. The presence of fungi as endophytes in sunflower tissues was confirmed by amplification of the region consisting of the 5.8S ribosomal DNA and internal transcribed spacers 1 and 2 using the primer set ITS5/ITS4 (White et al., 1990). Optimized PCR assays were carried out in a final volume of $25 \mu \mathrm{l}$ containing $0.4 \mu \mathrm{M}$ of each primer, $800 \mu \mathrm{M}$ dNTPs, $2.5 \mu 110 \times$ PCR buffer (800 mM Tris-HCl, $\mathrm{pH} 8.3-8.4$ at $25^{\circ} \mathrm{C}, 0.2 \%$ Tween $20 \mathrm{wt} / \mathrm{vol}$ ), 0.75 U Horse-Power Taq DNA polymerase (Canvax Biotech), $2.5 \mathrm{mM} \mathrm{MgCl}_{2}$, and $10 \mathrm{ng}$ fungal DNA. The following profile was set for the amplifications: 3 min initial denaturation at $95^{\circ} \mathrm{C} ; 30$ cycles of $30 \mathrm{~s}$ annealing at $56^{\circ} \mathrm{C}, 2 \mathrm{~min}$ of extension at $72{ }^{\circ} \mathrm{C}$ and $30 \mathrm{~s}$ denaturation at $95^{\circ} \mathrm{C}$; and a final extension step of $10 \mathrm{~min}$ at $72{ }^{\circ} \mathrm{C}$. Mycelial DNA of the fungi grown on PDA and sunflower DNA were used as positive controls, and water was used as a negative amplification control. All reactions were made in a $\mathrm{T} 1$ thermocycler (Whatman Biometra). Amplification products were separated by horizontal electrophoresis in 3.5\% agarose gels containing $0.05 \mu 1 / \mathrm{ml}$ GoodView nucleic acid stain (SBS Genetech Co., Ltd.) and visualized over a UV light 
source. A 100-2,000 bp BrightMAX DNA ladder (Canvax Biotech) was included in the electrophoresis.

\subsection{Data analysis}

All the experiments in this work were repeated once and, after assessing the lack of any significant differences between the two replicates (McIntosh, 1983), data were pooled and analysed using Statistix 10 (Analytical Software).

Data expressed as percentages (IMG and Ss) were transformed using an arcsine

transformation: $\mathrm{Y}=\operatorname{arcsine} \sqrt{\frac{\text { Variable }}{100}}$. Homoscedasticity (Brown and Forsythe test), normality (Shapiro-Wilk test) and randomization of residues (graphical test) were checked in order to perform an analysis of variance (ANOVA) of transformed IMG (dual culture experiments) and transformed Ss and AUDPC (greenhouse experiment). Dual culture experiments, as well as the greenhouse experiment, were statistically analysed according to completely randomized factorial designs. When significances were found for main factors and/or for their double interaction, means were compared using Fisher's least significant difference (LSD) test $(\alpha=.05)$.

\section{Results}

\subsection{Dual cultures}

When cultured together with the sunflower pathogens, all the EF strains showed an antagonistic activity, as they significantly inhibited the mycelial growth of both $V$. dahliae ( $p$ $<.001)$ and $C$. helianthi $(p<.0001)$. Furthermore, in dual cultures involving $V$. dahliae, the mycelial growth of the pathogen was significantly dependent on the $\operatorname{EF}(p<.0001)$ and the 
pathogen isolate $(p<.001)$, although the interaction $\mathrm{EF} \times$ pathogen isolate was not significant $(p=.1761)$. The percentage of IMG of $V$. dahliae ranged from $8.3 \%$ ( $V$. dahliae VdS0216 cocultured with EABb 04/01-Tip strain of B. bassiana) to $63.5 \%$ (V. dahliae VdS1016 cocultured with EABb 01/33-Su strain of B. bassiana). Additionally, M. brunneum EAMb 01/158-Su presented the lowest IMG for four of the six $V$. dahliae isolates, whereas $B$. bassiana $\mathrm{EABb}$ 01/33-Su gave the highest inhibition against four of the six isolates (Table 2). Furthermore, EABb 01/33-Su strain caused an average IMG of $47.0 \%$ on the six $V$. dahliae isolates, EAMa 01/58-Su of 42.5\%, EAMb 09/01-Su of 40.9\%, EABb 04/01-Tip of 30.6\%, and EAMb 01/158-Su of 24.3\%. Lastly, the three M. brunneum strains caused an average IMG of $35.7 \%$ on the six $V$. dahliae isolates, whereas the two B. bassiana strains caused an average IMG of $38.6 \%$.

In the case of $C$. helianthi, the EF significantly affected the pathogen's mycelial growth $(p<.0001)$, which was not dependent on $C$. helianthi isolate $(p=.9808)$; nor was the interaction between them significant $(p=.3386)$. The IMG of $C$. helianthi ranged from $19.6 \%$ (EAMb 01/158-Su of M. brunneum) to $37.4 \%$ (EABb 01/33-Su of B. bassiana) (Table 3). The three M. brunneum strains caused an average IMG of $24.5 \%$ on the three $C$. helianthi isolates, whereas the two B. bassiana strains caused an average IMG of $34.8 \%$.

Finally, inhibition halos were observed when M. brunneum strain EAMb 01/158-Su was co-cultured with any of the pathogens. In addition, the EF strains EAMb 09/01-Su and EABb 01/33-Su were able to overgrow the mycelium of the pathogens. The EF strain EAMa 01/58-Su was able to facultatively cause inhibition halos and overgrow the pathogens. Types of antagonism are shown in Figure 1: inhibition of $V$. dahliae and/or C. helianthi by $M$. brunneum (Figure 1a,c) and overgrowth of B. bassiana on $V$. dahliae and/or C. helianthi (Figure 1b,d). 


\subsection{Hyphal interaction}

When slides of dual cultures were cut from the culture medium and observed under the microscope, with the exception of the lack of contact between EAMb 01/158-Su strain and either of the pathogens, contact between hyphae of $V$. dahliae or hyphae of $C$. helianthi and all the other EF strains was observed (Figure S1). No antagonistic interactions were observed.

\subsection{Greenhouse experiment}

Control plants not inoculated with $V$. dahliae did not show any symptoms of verticillium wilt and were excluded from data analysis. Initial symptoms of verticillium wilt were observed in the control plants inoculated with the pathogen 1 week after inoculation, and they reached 95\% Ss at the end of the experiment (Figure 2a). Significant reductions in both Ss and AUDPC due to $V$. dahliae were associated with treatments with one strain of each EF species: M. brunneum EAMb 09/01-Su (53\% and 1096, respectively) and B. bassiana EABb 01/33-Su (31\% and 475, respectively) compared with the control plants (95\% and 2226 , respectively). The two remaining EF strains did not have a significant effect on verticillium wilt (either disease severity or AUDPC) (Figure 2a,b).

In the first assessment of the population density of the EF isolates, the cfu/g substrate varied between $8.25 \times 10^{5}(\mathrm{EABb} 01 / 33-\mathrm{Su})$ and $1.2 \times 10^{5}(\mathrm{EABb} 04 / 01-\mathrm{Tip})$ at the moment of inoculation with $V$. dahliae (Figure 3a). Four weeks later, these populations decreased down to $3.75 \times 10^{4}(\mathrm{EABb} 01 / 33-\mathrm{Su})$ and 0 (EABb 04/01-Tip). All the strains showed a marked decrease in populations 1 week after inoculation with $V$. dahliae, with the exception of EABb 04/01-Tip, which showed very low levels throughout the five sampling weeks, and decreased drastically during the last week (Figure 3a). In contrast, the severity of wilt symptoms in plants treated with EF and later on inoculated with $V$. dahliae increased mainly 
between the first and second week, and varied little until the end of the experiment (Figure $3 b)$.

\subsection{Microbiological and molecular detection of $V$. dahliae and entomopathogenic fungi in sunflower}

At the end of the experiment, $V$. dahliae was successfully isolated from those plants that had been inoculated with the pathogen irrespective of the treatment with EF. The isolation percentages ranged from $32 \%$ to $64 \%$ in the treatments with EABb $01 / 33-\mathrm{Su}$ and $\mathrm{EAMb}$ 09/01-Su, respectively (Table 4). Lastly, none of the EF was successfully isolated from sunflower leaves at the end of the experiment.

With regard to the molecular analyses, amplifications of samples from plants inoculated with $V$. dahliae yielded a $550 \mathrm{bp}$ fragment, which is diagnostic of the pathogen (Figure 4a). However, we were unable to confirm the presence of any of the EF strains in sunflowers inoculated with $V$. dahliae. The most interesting finding was that, in the absence of $V$. dahliae, bands of similar sizes to those of B. bassiana and M. brunneum were amplified, showing that the four EF strains succeed in endophytically colonizing the plants (Figure 4b). As expected, all the samples yielded the $750 \mathrm{bp}$ band diagnostic of sunflower (Figure 4a,b).

\section{Discussion}

In this work, we assessed the performance of five strains of EF as biological control agents against the sunflower pathogens $V$. dahliae and $C$. helianthi. Our results show that species of Metarhizium and Beauveria can play an active role as antagonists of those pathogens. Both the EF strain and the $V$. dahliae isolate had a significant effect on the mycelial growth of the pathogen when co-cultured with the EF, whereas under the same experimental conditions, the 
growth of $C$. helianthi was only dependent on the EF strain. This is not surprising, as $V$. dahliae affecting sunflowers in Europe exhibits a wide diversity that is highly dependent on its geographical origin (Martin-Sanz et al., 2018b). As for C. helianthi, it was recently reported as being a pathogen of sunflower (Crous et al., 2019). Although species diversity information is still needed, heterogeneity was not expected within just the three isolates included in our experiments. The most revealing finding was that, in spite of EF inhibiting the growth of both pathogens, no trend in their effect was observed as being associated with either genus (B. bassiana or M. brunneum), and one B. bassiana strain was the most effective one (EABb 01/33-Su) against $C$. helianthi and most of the $V$. dahliae isolates. Similarly, the antagonism of EF against the olive pathogens $V$. dahliae, Phytophthora megasperma and $P$. inundata was largely dependent on the particular strain more than on any other factor (Lozano-Tovar et al., 2013).

From the results of the dual culture experiments and the microscope observations, we identified two types of antagonism exerted by EF against plant pathogenic fungi. First, mycelial growth inhibition of the pathogens by two strains of $B$. bassiana (EAMb 09/01-Su and $\mathrm{EABb} 01 / 33-\mathrm{Su}$ ) and one of M. brunneum (EAMa 01/58-Su) was associated with overgrowth of the EF on both $V$. dahliae and $C$. helianthi. Not only do our EF strains have notoriously high growth rates (Quesada-Moraga et al., 2014; Raya-Diaz et al., 2017a; 2017b), but the ability of M. brunneum strain EAMa 01/58-Su to grow over the mycelia of olive root rot pathogens has already been reported (Lozano-Tovar et al., 2013). Similarly, Varo et al. (2016) carried out dual cultures to test the efficacy of several biological control agents against an isolate of $V$. dahliae that was pathogenic to olive. They identified two modes of antagonism: clear inhibition zones without $V$. dahliae mycelium, and the growth of the biocontrol agents over the pathogen. In our microscopy work, we observed contact and even intertwining of the hyphae of the confronted fungi in the absence of any mechanical 
alteration or degradation, which is in agreement with competition as being the mode of action of M. brunneum and B. bassiana. Other authors have also proposed competition as at least one of the modes of action operating in disease suppression by EF (Ownley et al., 2010; Lozano-Tovar et al., 2013). Secondly, the presence of highly marked inhibition zones when EAMb 01/158-Su strain of M. brunneum was dually plated with the pathogens, and hyphae that were clearly distant from those of either $V$. dahliae or $C$. helianthi under the microscope, suggest that the detrimental effect of the strain is associated with the release of diffusible inhibitory substances. An antibiotic effect of M. brunneum against olive pathogens has already been reported by Lozano-Tovar et al. (2017). The chemical identification of compounds produced by EAMb 01/158-Su strain displaying antibiosis in our experiments is the subject of further work.

Regarding the development of verticillium wilt in sunflowers treated with B. bassiana or M. brunneum (strains EABb 01/33-Su and EAMb 09/01-Su, respectively) and thereafter inoculated with the pathogen, both entomopathogens were associated with significant disease reductions, with $B$. bassiana (EABb 01/33-Su) having the most pronounced effect. Although none of the EF strains were detected inside the plants in the presence of $V$. dahliae, an outstanding finding was that, in the absence of the pathogen, the four EF strains succeeded in establishing themselves in sunflowers. Despite these EF strains being well known as transient colonizers of different hosts when applied by foliar spraying (Resquín-Romero et al., 2016; Garrido-Jurado et al., 2017), in our work, with soil drenching applications, they were not only able to survive and settle in the substrate, but also to penetrate the plants and establish themselves as endophytes for weeks. Even though the entomopathogens were unable to compete against $V$. dahliae inside sunflowers, their ability to colonize the plants is an essential feature that could be useful against sunflower pathogens other than $V$. dahliae or even as plant growth promoters (Ownley et al., 2010). Also, the protection conferred by 
entomopathogenic fungi against verticillium wilt might be due, at least for the most part, to the direct competition between $V$. dahliae and M. brunneum (EAMb 09/01-Su) or B. bassiana $(\mathrm{EABb} 01 / 33-\mathrm{Su})$ in the soil. Both of these strains are highly adapted to the soil environment (Garrido-Jurado et al., 2017), which seems to favour their competition with $V$. dahliae. Varo et al. (2016) tested a wide variety of microorganisms against verticillium wilt in olive, reporting that both the antagonism in the soil/plant and systemic mechanisms could contribute to a relief of symptoms, thus controlling the disease. In this respect, Raya-Diaz et al. (2017a) reported that EF could promote sunflower growth and inflorescence production under controlled conditions due to an enhancement in Fe bioavailability. Whether this could be related to some extent to the triggering of systemic resistance in sunflower, consequently having a role in disease control, should be explored in future research.

In accordance with Raya-Diaz et al. (2017b), who determined that soil treatment was the best option for the application of EF because it resulted in good population recovery levels, we found that the four EF strains dramatically decreased during the first 4 weeks in the soil (from treatment to inoculation) and, thereafter, during the following 4 weeks. One of the strains (EABb 04/01-Tip) was even unable to remain in the soil as a stable population. Moreover, and as mentioned above, the persistence of EABb 01/33-Su and EAMb 09/01-Su populations in the soil could be the consequence of their adaptation to this environment and a first requirement for controlling verticillium wilt. Also, the transient colonization of melon plants by these same strains after foliar applications has been reported (Garrido-Jurado et al., 2017), but this does not seem to be the operating mechanism in our experiment in which treatments were conducted by soil drenching. Although present in the soil, none of the three strains was reisolated from plant samples, suggesting that the observed in vivo effect of EF against $V$. dahliae is not plant-located, but is most likely the consequence of the intense competition waged by B. bassiana (EABb 01/33-Su) and/or M. brunneum (EAMb 09/01-Su) 
against the pathogen in the soil, as already mentioned. Moreover, as a result of the profuse growth of these EFs in comparison to that of $V$. dahliae, pathogen soil populations might be decreased, and root penetration and the development of eventual symptoms delayed in time, as suggested by the positive identification of $V$. dahliae in plants treated with the EF. In addition to the soil-mediated interplay between EABb 01/33-Su or EAMb 09/01-Su and $V$. dahliae suggested by the results of this work, further research should explore whether these strains are able to penetrate sunflower roots and inhibit $V$. dahliae in the host.

Few studies relating EF and insect pests of sunflower have been carried out so far. One by Takov et al. (2013) reported the presence of $B$. bassiana associated with a sunflower crop in Bulgaria. According to the authors, the indigenous EF caused high mortality rates in the sunflower pest Tanymecus dilaticollis. Similarly, Barker (1999) tested the efficacy of Metarhizium anisopliae (Metsch) and B. bassiana against the sunflower pest Cochylis hospes under laboratory conditions. In spite of the high efficacy in controlling sunflower insect pests reported in both works, the long-term survival of EF was also pointed out as being a priority for potential field applications.

In conclusion, both M. brunneum and B. bassiana are able to inhibit the mycelial growth of $V$. dahliae and $C$. helianthi and, according to the dual culture and microscopy results, two types of antagonism can operate: competition and/or antibiosis. In addition, although the entomopathogens were not found inside the plants in the presence of $V$. dahliae, they were when the pathogen was absent. In fact, they were able to establish themselves inside sunflowers, suggesting their potential utility in applications such as, for instance, those to control sunflower diseases other than verticillium wilt, or to promote sunflower growth. Finally, the ability of EF populations to persist in the substrate for several weeks, together with disease reduction observed in treatments with some strains, suggests that the protection 
they confer against verticillium wilt might not be plant-located, but is more likely to be the consequence of their competition with $V$. dahliae in the soil.

\section{Acknowledgements}

This research was partially supported by the Spanish Ministry of Economy and Competitiveness project AGL2016-80483-R to Professor E. Quesada-Moraga and the Spanish National Research Council project PIE201940E058 to L.M.R., and grant FPU16/03983 from the Ministry of Education, Culture and Sports (Spain) to P.M.F. We thank Professor E. Quesada-Moraga for providing the entomopathogenic fungi used in this work.

\section{Conflict of interests}

The authors declare that they have no competing interests.

\section{Data availability statement}

The data that support the findings of this study are available from the authors upon reasonable request.

\section{References}

Barker, J.F. (1999) Laboratory evaluation of the pathogenicity of Beauveria bassiana and Metarhizium anisopliae to larvae of the banded sunflower moth, Cochylis hospes 
(Lepidoptera: Cochylidae). Great Lakes Entomology, 32, 101-106.

Campbell, C.L. and Madden, L.V. (1990) Introduction to Plant Disease Epidemiology. New York, USA: John Wiley and Sons.

Crous, P.W., Carnegie, A.J., Wingfield, M.J., Sharma, R., Mughini, G., Noodeloos, M.E. et al. (2019) Fungal Planet description sheets: 868-950. Persoonia, 42, 291-473.

Erreguerena, I.A., Rojo, R., Quiroz, F.J., Clemente, G.E., and Escande, A.R. (2019)

Relationship between Verticillium dahliae inoculum and sunflower wilt in Argentina. Canadian Journal of Plant Pathology, 41, 576-584.

FAOSTAT (2020) Food and Agriculture Data. Available at: http://www.fao.org/faostat/en/\#home [Accessed 25 March 2020].

de Faria, M.R. and Wraight, S.P. (2007) Mycoinsecticides and Mycoacaricides: A comprehensive list with worldwide coverage and international classification of formulation types. Biological Control, 43, 237-256.

Fick, G.N. and Zimmer, D.E. (1974) Monogenic Resistance to Verticillium Wilt in Sunflowers. Fargo, ND, USA: Agricultural Experiment Station, North Dakota State University. Journal paper No. 518.

Garcia-Carneros, A.B., Garcia-Ruiz, R. and Molinero-Ruiz, L. (2014) Genetic and molecular approach to Verticillium dahliae infecting sunflower. Helia, 37, 205-214.

Garcia-Ruiz, R., Garcia-Carneros, A.B. and Molinero-Ruiz, L. (2014) A new race of Verticillium dahliae causing leaf mottle of sunflower in Europe. Plant Disease, 98, 1435. 
Garrido-Jurado, I., Fernandez-Bravo, M., Campos, C. and Quesada-Moraga, E. (2015)

Diversity of entomopathogenic Hypocreales in soil and phylloplanes of five

Mediterranean cropping systems. Journal of Invertebrate Pathology, 130, 97-106.

Garrido-Jurado, I., Resquin-Romero, G., Amarilla, S.P., Rios-Moreno, A., Carrasco, L. and Quesada-Moraga, E. (2017) Transient endophytic colonization of melon plants by entomopathogenic fungi after foliar application for the control of Bemisia tabaci Gennadius (Hemiptera: Aleyrodidae). Journal of Pest Science, 90, 319-330.

Gonzalez-Fernandez, S. (2015) Verticillium dahliae Patógeno de Girasol: Control Mediante Fuentes de Resistencia y Caracterización Molecular de una Colección Internacional. Master's Thesis. Andalusia, Spain: University of Cordoba and University of Cadiz.

Gonzalez-Mas, N., Quesada-Moraga, E., Plaza, M., Fereres, A. and Moreno, A. (2019) Changes in feeding behaviour are not related to the reduction in the transmission rate of plant viruses by Aphis gossypii (Homoptera: Aphididae) to melon plants colonized by B. bassiana (Ascomycota: Hypocreales). Biological Control, 130, 95-103.

Gulya, T. (2007) New strain of Verticillium dahliae in North America. Helia, 30, 115-120.

Gulya, T.J., Rashid, K.Y. and Marisevic, S.M. (1997) Sunflower diseases. In: Schneiter, A.A. (Ed.) Sunflower Technology and Production. Madison, Wisconsin, USA: ASA, CSSA, SSSA Publications, pp. 263-379.

Gulya, T., Harveson R., Mathew, F., Block, C. Thompson, S., Kandel, H. et al. (2019) Comprehensive disease survey of U.S. sunflower: disease trends, research priorities and unanticipated impacts. Plant Disease, 103, 601-618.

Hoagland, D.R. and Arnon, D.I. (1950) The water-culture method for growing plants without 
soil. California Agricultural Experiment Station, 347, p. 32.

Keyser, C.A., Jensen, B. and Meyling, N.V. (2016) Dual effects of Metarhizium spp. and Clonostachys rosea against an insect and a seed-borne pathogen in wheat. Pest Management Science, 72, 517-526.

Klosterman, S.J., Atallah, Z.K., Vallad, G.E. and Subbarao, K.V. (2009) Diversity, pathogenicity, and management of verticillium species. Annual Review of Phytopathology, 47, 39-62.

Lozano-Tovar, M.D., Ortiz-Urquiza, A., Garrido-Jurado, I., Trapero-Casas, A. and QuesadaMoraga, E. (2013) Assessment of entomopathogenic fungi and their extracts against a soil-dwelling pest and soil-borne pathogens of olive. Biological Control, 67, 409-420.

Lozano-Tovar, M.D., Garrido-Jurado, I., Quesada-Moraga, E., Raya-Ortega, M.C. and Trapero-Casas, A. (2017) Metarhizium brunneum and Beauveria bassiana release secondary metabolites with antagonistic activity against Verticillium dahliae and Phytophthora megasperma olive pathogens. Crop Protection, 100, 186-195.

Martin-Sanz, A., Rueda, S., Garcia-Carneros, A.B. and Molinero-Ruiz, L. (2018a) Cadophora malorum: A new pathogen of sunflower causing wilting, yellowing, and leaf necrosis in Russia. Plant Disease, 102, 823.

Martin-Sanz, A., Rueda, S., Garcia-Carneros, A.B., Gonzalez-Fernandez, S., MirandaFuentes, P., Castuera-Santacruz, S. et al. (2018b) Genetics, host range, and molecular and pathogenic characterization of Verticillium dahliae from sunflower reveal two differentiated groups in Europe. Frontiers in Plant Science, 9, 288.

McIntosh, M.S. (1983) Analysis of combined experiments. Agronomy Journal, 75, 153-155. 
Molinero-Ruiz, L. (2019) Recent advances on the characterization and control of sunflower soilborne pathogens under climate change conditions. $O C L, 26,2$.

Molinero-Ruiz, M.L., Melero-Vara, J.M., Gulya, T.J. and Dominguez, J. (2003) First report of resistance to metalaxyl in downy mildew of sunflower caused by Plasmopara halstedii in Spain. Plant Disease, 87, 749.

Molinero-Ruiz, M.L., Cordon-Torres, M.M., Martinez-Aguilar, J., Melero-Vara, J.M. and Dominguez, J. (2008) Resistance to metalaxyl and to metalaxyl-M in populations of Plasmopara halstedii causing downy mildew in sunflower. Canadian Journal of Plant Pathology, 30, 97-105.

Ownley, B.H., Gwinn, K.D. and Vega, F.E. (2010) Endophytic fungal entomopathogens with activity against plant pathogens: ecology and evolution. BioControl, 55, 113-128.

Pegg, G.F. and Brady, B.L. (2002) Verticillium Wilts. Wallingford, UK: CAB International.

Putt, E.D. (1958) Note on resistance of sunflowers to leaf mottle disease. Canadian Journal of Plant Science, 38, 274-276.

Quesada-Moraga, E., Lopez-Diaz, C. and Landa, B.B. (2014) The hidden habit of the entomopathogenic fungus Beauveria bassiana: First demonstration of vertical plant transmission. PLoS ONE, 9, e89278.

Radi, S. and Gulya, T. (2007) Sources of resistance to a new strain of Verticillium dahliae on sunflower in North America - 2006. In: Proceedings of the 29th Sunflower Research Workshop. Bismarck, North Dakota, USA: National Sunflower Association, pp. 10-11.

Raya-Diaz, S., Quesada-Moraga, E., Barron, V., del Campillo, M.C. and Sanchez-Rodriguez, A.R. (2017a) Redefining the dose of the entomopathogenic fungus Metarhizium 
brunneum (Ascomycota, Hypocreales) to increase Fe bioavailability and promote plant growth in calcareous and sandy soils. Plant and Soil, 418, 387-404.

Raya-Diaz, S., Sanchez-Rodriguez, A.R., Segura-Fernandez, J.M., del Campillo, M.C. and Quesada-Moraga, E. (2017b) Entomopathogenic fungi-based mechanisms for improved Fe nutrition in sorghum plants grown on calcareous substrates. PLOS ONE, 12, e0185903.

Rekanovic, E., Milijasevic, S., Todorovic, B. and Potocnik, I. (2007) Possibilities of biological and chemical control of verticillium wilt in pepper. Phytoparasitica, 35, $436-441$.

Resquín-Romero, G., Garrido-Jurado, I., Delso, C., Ríos-Moreno, A. and Quesada-Moraga, E. (2016) Transient endophytic colonizations of plants improve the outcome of foliar applications of mycoinsecticides against chewing insects. Journal of Invertebrate Pathology, 136, 23-31.

Schneiter, A.A. and Miller, J.F. (1981) Description of sunflower stages. Crop Science, 21, 901-903.

Schulz, B., Boyle, C., Draeger, S., Rommert, A.K. and Krohn, K. (2002) Endophytic fungi: a source of novel biologically active secondary metabolites. Mycological Research, 106, 996-1004.

Shrivastava, G., Ownley, B.H., Auge, R.M., Toler, H., Dee, M., Vu, A., Kollner, T.G. and Chen, F. (2015) Colonizaton by arbuscular mycorrhizal and endophytic fungi enhanced terpene production in tomato plants and their defense against a herbivorous insect. Symbiosis, 65, 65-74. 
Takov, D.I., Draganova, S.A. and Toshova, T.B. (2013) Gregarine and Beauveria bassiana infections of the grey corn weevil, Tanymecus dilaticollis (Coleoptera: Curculionidae). Acta Phytopathologica et Entomologica Hungarica, 48, 309-319.

Varo, A., Raya-Ortega, M.C. and Trapero, A. (2016) Selection and evaluation of microorganisms for biocontrol of Verticillium dahliae in olive. Journal of Applied Microbiology, 121, 767-777.

White, T.J., Bruns, T., Lee, S. and Taylor, J. (1990) Amplification and direct sequencing of fungal ribosomal RNA genes for phylogenetics. In: Innis, M.A., Gelfand, D.H., Sninsky, J.J. and White, T.J. (Eds.) PCR Protocols: A Guide to Methods and Applications. New York, USA: Academic Press, pp. 315-322.

\section{Supporting Information}

Additional Supporting Information may be found in the online version of this article at the publisher's web-site.

Figure S1 Hyphal contact between an entomopathogenic fungus (Metarhizium brunneum or Beauveria bassiana) and Verticillium dahliae or Cadophora helianthi in dual cultures. (a) $M$. brunneum versus $V$. dahliae; (b) B. bassiana versus $V$. dahliae; (c) M. brunneum versus $C$. helianthi; (d) B. bassiana versus C. helianthi.

Figure legends

Figure 1 Antagonism exerted by entomopathogenic fungi Metarhizium brunneum and Beauveria bassiana on sunflower pathogens Verticillium dahliae and Cadophora helianthi in 
dual cultures. (a) Inhibition on $V$. dahliae VdS0216 by M. brunneum EAMa 01/58-Su; (b) overgrowth of B. bassiana EABb 01/33-Su on V. dahliae VdS0916; (c) inhibition on $C$. helianthi CadoSU01-17 by M. brunneum EAMb 01/158-Su; (d) overgrowth of B. bassiana EABb 01/33-Su on C. helianthi CadoSR02-16.

Figure 2 Verticillium wilt severity, expressed as (a) percentage of the foliar tissue affected (\%) 4 weeks after pathogen inoculation (64 days after sowing); and (b) area under disease progress curve (AUDPC) at the same time, in sunflower plants inoculated with Verticillium dahliae and treated with different entomopathogenic fungi. Control plants were inoculated with $V$. dahliae alone. Letters on the bars indicate homogeneous groups according to LSD post hoc test $(p<.05)$. Analyses of verticillium wilt severity (a) were performed on transformed data using an arcsine transformation.

Figure 3 (a) Populations of four entomopathogenic fungi recovered from the substrate of pots in which sunflower plants were grown. Inoculation of sunflower plants with Verticillium dahliae was performed in week 0 . Verticillium wilt symptoms were assessed between weeks 1 and 4. (b) Time course expression of verticillium wilt symptoms (severity of symptoms, \%) in EF-treated plants from weeks 1 to 4 after inoculation with $V$. dahliae. Vertical bars represent the severity of symptoms in inoculated plants averaged across EF treatments.

Figure 4 Band patterns obtained after PCR amplification of the ribosomal DNA region (5.8S rDNA and internal transcribed spacers 1 and 2) with the universal primer set ITS5 and ITS4 from individual sunflower plants. DNA samples were obtained from lyophilized stem tissue of sunflower plants (breeding line RHA801, R5 stage, 64 days after sowing) treated with: (a) four entomopathogenic fungi (EF) strains: Beauveria bassiana $\mathrm{EABb} 01 / 33-\mathrm{Su}$ and $\mathrm{EABb}$ 04/01-Tip, and Metarhizium brunneum EAMa 01/58-Su and EAMb 09/01-Su (always in the same order). Lanes 1-6, DNA from sunflower breeding line RHA801, $V$. dahliae isolate 
VdS0113, and the four EF strains; lanes 7-14, DNA from two sunflowers only treated with the four EF strains. (b) EF followed by inoculation with Verticillium dahliae isolate VdS0113. Lanes 1-5, DNA from sunflower, and the four EF strains; lanes 6-13, DNA from two sunflowers treated with the four EF strains and inoculated with $V$. dahliae 1 month later. M, 100-2,000 bp BrightMAX DNA ladder (Canvax Biotech). 


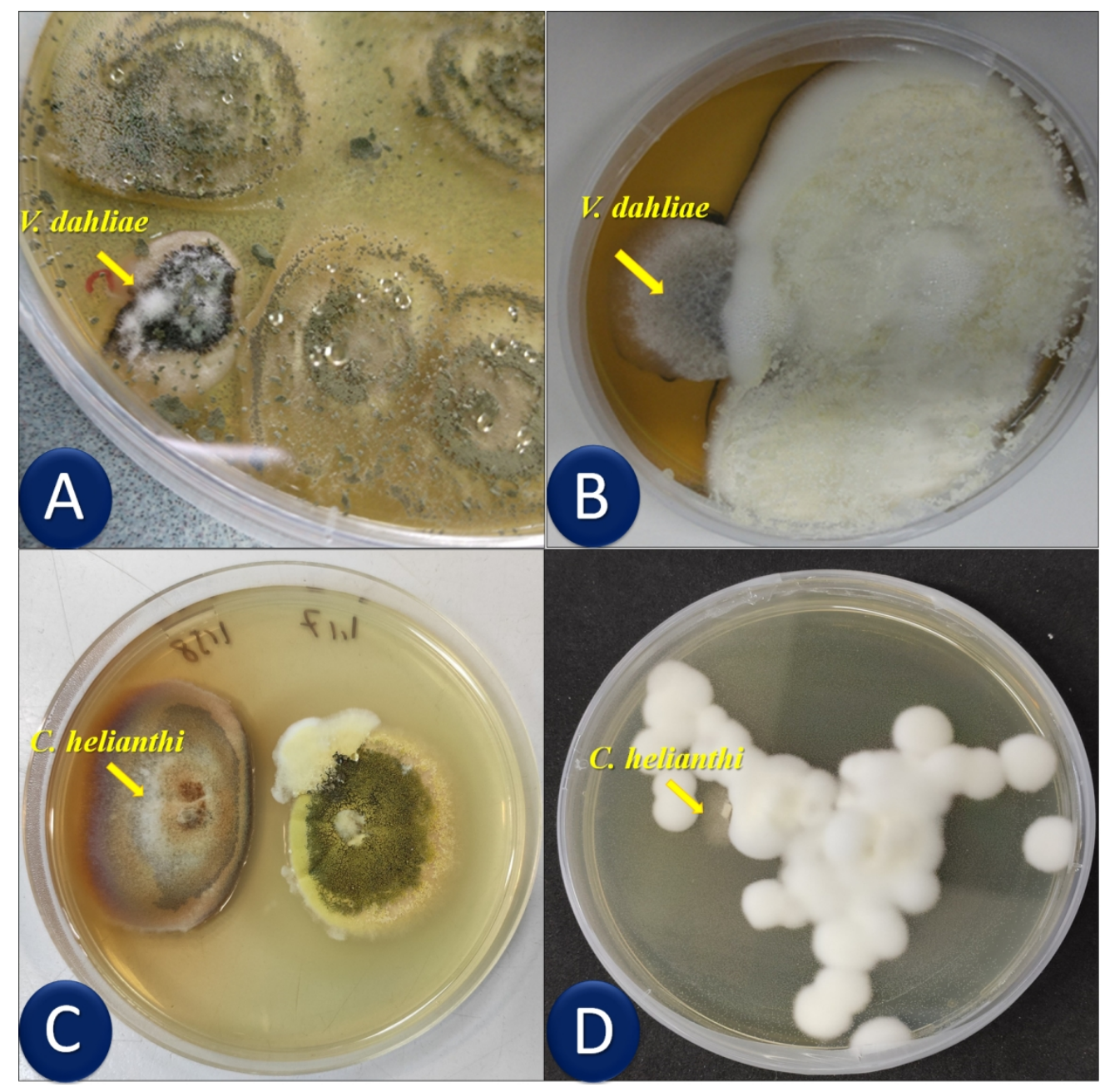

426x421mm (96 x 96 DPI) 
A
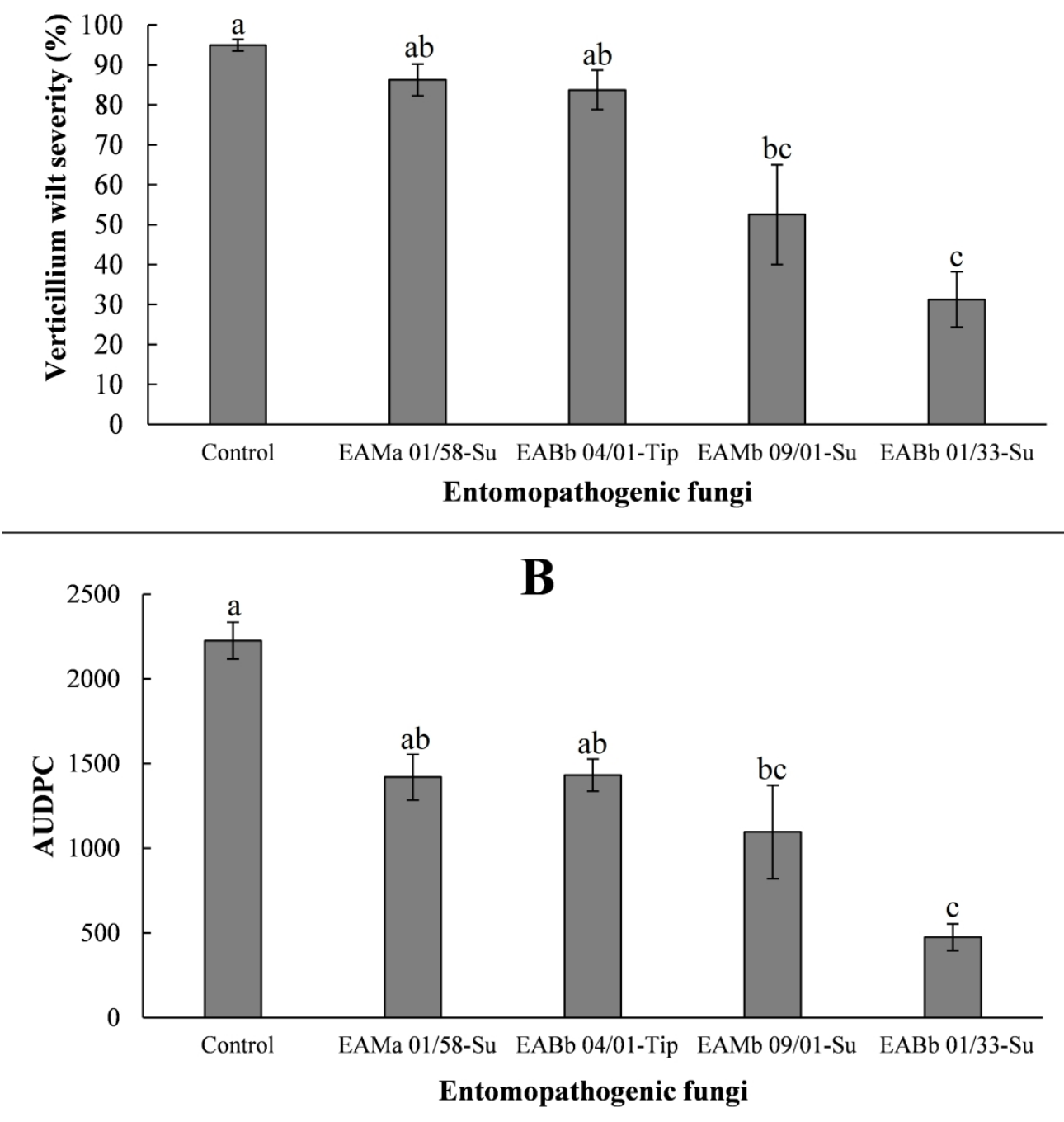

$886 \times 1024 \mathrm{~mm}(96 \times 96 \mathrm{DPI})$ 


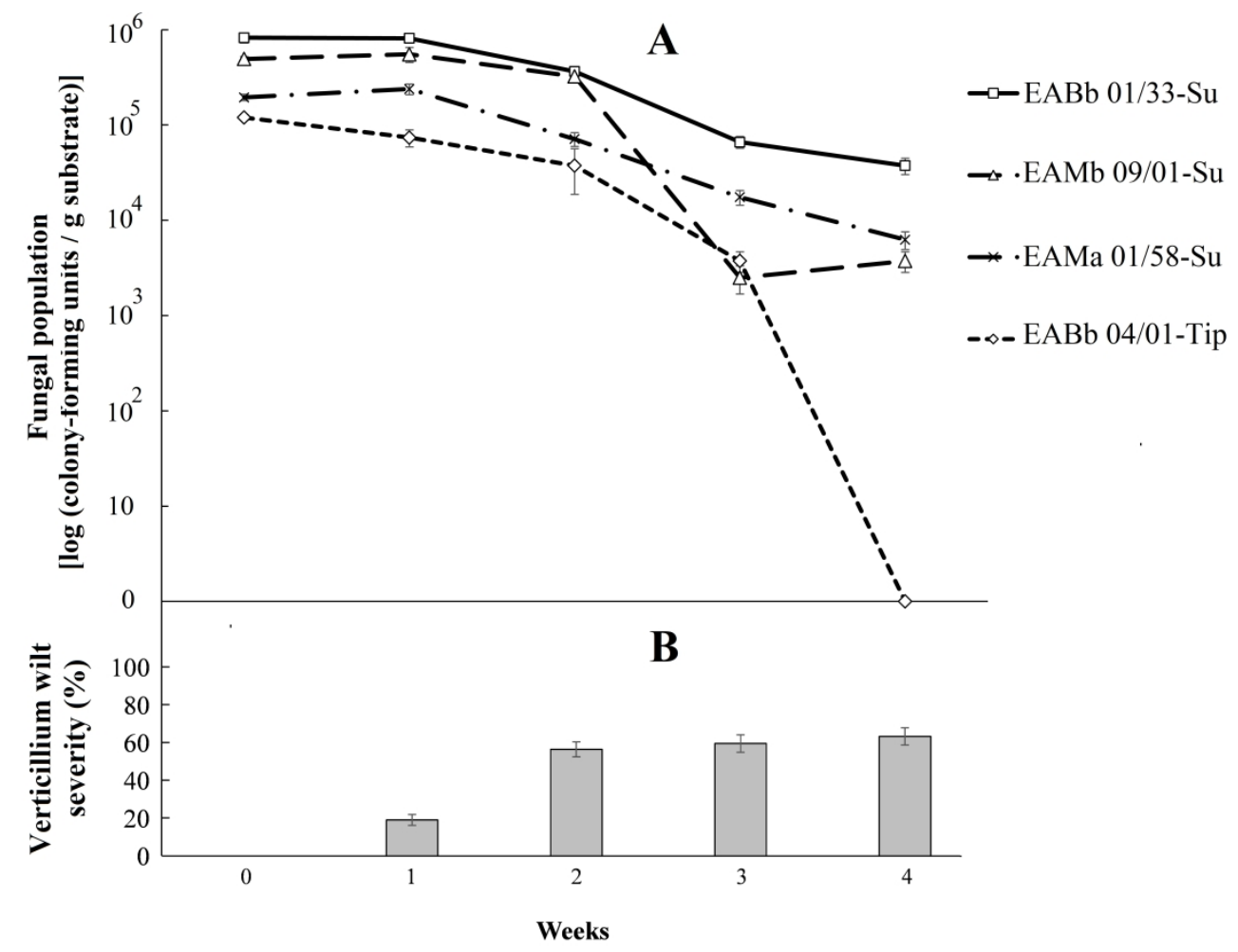

$1439 \times 1132 \mathrm{~mm}(96 \times 96$ DPI $)$ 


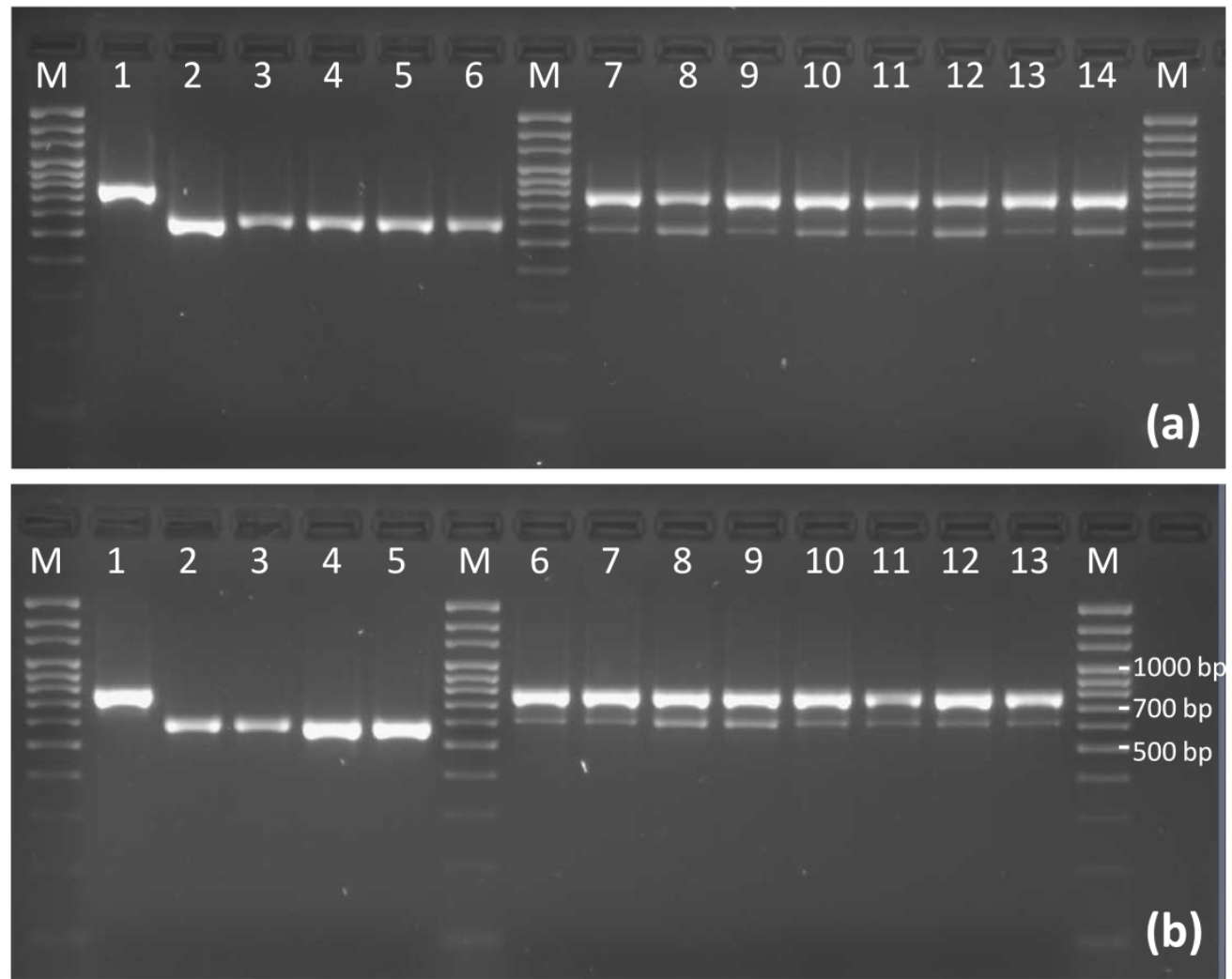

$745 \times 599 m m(96 \times 96$ DPI) 
Table 1 Fungal isolates used in this work, with a list of the experiments in which they were used

\begin{tabular}{|c|c|c|c|c|}
\hline $\begin{array}{l}\text { Fungal } \\
\text { species }\end{array}$ & Isolate & $\begin{array}{l}\text { Host of } \\
\text { isolation }\end{array}$ & Origin & Experiments \\
\hline \multirow[t]{7}{*}{$\begin{array}{l}\text { Verticillium } \\
\text { dahliae }\end{array}$} & VdS0113 & Sunflower & Cadiz, Spain & $\begin{array}{l}\text { Greenhouse } \\
\text { experiment }\end{array}$ \\
\hline & VdS1014 & Sunflower & $\begin{array}{l}\text { Valu lui Traian, } \\
\text { Romania }\end{array}$ & Dual cultures \\
\hline & VdS0216 & Sunflower & Montech, France & Dual cultures \\
\hline & VdS0316 & Sunflower & Macerata, Italy & Dual cultures \\
\hline & VdS0616 & Sunflower & $\begin{array}{l}\text { Slava Rusa, } \\
\text { Romania }\end{array}$ & Dual cultures \\
\hline & VdS0916 & Sunflower & $\begin{array}{l}\text { Kastamonu, } \\
\text { Ahmetbey, } \\
\text { Turkey }\end{array}$ & Dual cultures \\
\hline & VdS1016 & Sunflower & $\begin{array}{l}\text { Manvelivka, } \\
\text { Dnepropet, } \\
\text { Ukraine }\end{array}$ & Dual cultures \\
\hline \multirow[t]{3}{*}{$\begin{array}{l}\text { Cadophora } \\
\text { helianthi }\end{array}$} & $\begin{array}{l}\text { CadoSR02- } \\
16\end{array}$ & Sunflower & $\begin{array}{l}\text { Orenburg, } \\
\text { Russia }\end{array}$ & Dual cultures \\
\hline & $\begin{array}{l}\text { CadoSR03- } \\
16\end{array}$ & Sunflower & $\begin{array}{l}\text { Orenburg, } \\
\text { Russia }\end{array}$ & Dual cultures \\
\hline & $\begin{array}{l}\text { CadoSU01- } \\
17\end{array}$ & Sunflower & Kiev, Ukraine & Dual cultures \\
\hline \multirow[t]{3}{*}{$\begin{array}{l}\text { Metarhizium } \\
\text { brunneum }\end{array}$} & $\begin{array}{l}\text { EAMa } \\
01 / 58-\mathrm{Su}\end{array}$ & Wheat & Cordoba, Spain & $\begin{array}{l}\text { Greenhouse } \\
\text { experiment, dual } \\
\text { cultures }\end{array}$ \\
\hline & $\begin{array}{l}\text { EAMb } \\
01 / 158-\mathrm{Su}\end{array}$ & Olive & Seville, Spain & Dual cultures \\
\hline & $\begin{array}{l}\text { EAMb } \\
09 / 01-\mathrm{Su}\end{array}$ & Wheat & Seville, Spain & $\begin{array}{l}\text { Greenhouse } \\
\text { experiment, dual } \\
\text { cultures }\end{array}$ \\
\hline
\end{tabular}




\begin{tabular}{lllll}
$\begin{array}{l}\text { Beauveria } \\
\text { bassiana }\end{array}$ & EABb & Olive & Cadiz, Spain & $\begin{array}{l}\text { Greenhouse } \\
\text { experiment, dual } \\
\text { c1/33-Su }\end{array}$ \\
& & & cultures \\
& EABb & Iraella & Seville, Spain & Greenhouse \\
04/01-Tip & luteipes & & experiment, dual \\
& & & cultures \\
\hline
\end{tabular}


Table 2 Effect of different strains of the entomopathogenic fungi (EF) Metarhizium brunneum and Beauveria bassiana on the vegetative growth of Verticillium dahliae, expressed as inhibition of the mycelial growth (IMG)

\begin{tabular}{|c|c|c|c|}
\hline Isolate & EF & EF strain & IMG $(\%)^{\mathrm{a}}$ \\
\hline \multirow[t]{5}{*}{ VdS1014 } & M. brunneum & EAMa 01/58-Su & $53.8 \pm 7.0 \mathrm{a}$ \\
\hline & & EAMb 01/158-Su & $27.0 \pm 9.2 \mathrm{~b}$ \\
\hline & & EAMb 09/01-Su & $53.9 \pm 6.7 \mathrm{a}$ \\
\hline & B. bassiana & $\mathrm{EABb} 01 / 33-\mathrm{Su}$ & $45.6 \pm 6.8 \mathrm{ab}$ \\
\hline & & EABb 04/01-Tip & $41.8 \pm 9.4 \mathrm{ab}$ \\
\hline \multirow[t]{5}{*}{ VdS0216 } & M. brunneum & EAMa 01/58-Su & $37.8 \pm 10.1 \mathrm{a}$ \\
\hline & & EAMb 01/158-Su & $18.7 \pm 5.9 \mathrm{ab}$ \\
\hline & & EAMb 09/01-Su & $23.9 \pm 5.2 \mathrm{ab}$ \\
\hline & B. bassiana & EABb 01/33-Su & $39.5 \pm 10.2 \mathrm{a}$ \\
\hline & & EABb 04/01-Tip & $8.3 \pm 4.3 \mathrm{~b}$ \\
\hline \multirow[t]{5}{*}{ VdS0316 } & M. brunneum & EAMa 01/58-Su & $38.7 \pm 1.9 \mathrm{ab}$ \\
\hline & & EAMb 01/158-Su & $23.4 \pm 3.3 b$ \\
\hline & & EAMb 09/01-Su & $38.7 \pm 7.2 \mathrm{ab}$ \\
\hline & B. bassiana & $\mathrm{EABb} 01 / 33-\mathrm{Su}$ & $41.1 \pm 5.7 \mathrm{a}$ \\
\hline & & EABb 04/01-Tip & $29.9 \pm 5.9 \mathrm{ab}$ \\
\hline \multirow[t]{5}{*}{ VdS0616 } & M. brunneum & EAMa 01/58-Su & $44.0 \pm 6.4 \mathrm{a}$ \\
\hline & & EAMb 01/158-Su & $28.8 \pm 6.7 \mathrm{ab}$ \\
\hline & & EAMb 09/01-Su & $43.9 \pm 6.1 \mathrm{a}$ \\
\hline & B. bassiana & $\mathrm{EABb} 01 / 33-\mathrm{Su}$ & $42.9 \pm 11.4 \mathrm{ab}$ \\
\hline & & EABb 04/01-Tip & $22.0 \pm 5.8 \mathrm{~b}$ \\
\hline \multirow[t]{5}{*}{ VdS0916 } & M. brunneum & EAMa 01/58-Su & $33.8 \pm 6.6 b$ \\
\hline & & EAMb 01/158-Su & $24.3 \pm 5.4 \mathrm{~b}$ \\
\hline & & EAMb 09/01-Su & $46.1 \pm 12.3 \mathrm{ab}$ \\
\hline & B. bassiana & EABb 01/33-Su & $57.2 \pm 6.5 \mathrm{a}$ \\
\hline & & EABb 04/01-Tip & $55.6 \pm 3.5 \mathrm{a}$ \\
\hline \multirow[t]{4}{*}{ VdS1016 } & M. brunneum & EAMa 01/58-Su & $47.5 \pm 5.9 \mathrm{ab}$ \\
\hline & & EAMb 01/158-Su & $23.4 \pm 6.9 \mathrm{c}$ \\
\hline & & EAMb 09/01-Su & $34.8 \pm 5.6 \mathrm{bc}$ \\
\hline & B. bassiana & EABb 01/33-Su & $63.5 \pm 8.2 \mathrm{a}$ \\
\hline
\end{tabular}




\section{EABb 04/01-Tip $\quad 36.4 \pm 9.7$ bc}

Note. Plates were incubated at $25^{\circ} \mathrm{C}$ in the dark for 37 days, the time needed for the control of each $V$. dahliae isolate to reach the edge of the plate. For each $V$. dahliae isolate, means with a common letter are not significantly different according to Fisher's least significant difference test. Analyses were performed on transformed data using an acrsine transformation.

${ }^{\mathrm{a}}$ Mean $\pm S E$ of three replications of IMG expressed as percentages. 
Table 3 Effect of different strains of the entomopathogenic fungi (EF) Metarhizium brunneum and Beauveria bassiana on the vegetative growth of three isolates of Cadophora helianthi, expressed as inhibition of the mycelial growth (IMG)

\begin{tabular}{lll}
\hline EF & EF strain & IMG (\%) $^{\mathbf{a}}$ \\
\hline M. brunneum & EAMa 01/58-Su & $30.4 \pm 3.2 \mathrm{ab}$ \\
& EAMb 01/158-Su & $19.6 \pm 2.9 \mathrm{c}$ \\
& EAMb 09/01-Su & $23.5 \pm 3.0 \mathrm{bc}$ \\
B. bassiana & EABb 01/33-Su & $37.4 \pm 3.1 \mathrm{a}$ \\
& EABb 04/01-Tip & $32.2 \pm 2.7 \mathrm{a}$
\end{tabular}

Plates were incubated at $25^{\circ} \mathrm{C}$ in the dark for 37 days, the time needed for the control of each C. helianthi isolate to reach the edge of the plate. Means with a common letter are not significantly different according to Fisher's least significant difference test. Analyses were performed on transformed data using an acrsine transformation.

${ }^{\mathrm{a}}$ Mean $\pm S E$ of four replications of IMG expressed as percentages. 
Table 4 Isolation of Verticillium dahliae (\%) from sunflower plants inoculated with $V$. dahliae only (control) or with $V$. dahliae and one strain of entomopathogenic fungi, four weeks after inoculation with the pathogen

\begin{tabular}{ll}
\hline Strain & Isolation of $\boldsymbol{V}$.dahliae $\mathbf{( \% )}$ \\
\hline Control plants & $60.7 \pm 9.0$ \\
EABb 01/33-Su & $32.1 \pm 6.8$ \\
EABb 04/01-Tip & $42.9 \pm 14.3$ \\
EAMb 09/01-Su & $64.3 \pm 9.2$ \\
EAMa 01/58-Su & $46.4 \pm 14.7$ \\
\hline
\end{tabular}

Note. Data show mean $\pm S E$. 